\title{
Framework of Closed-loop Optimization System for Operation Management in Plant-wide Production Process
}

\author{
Changxin Liü, a \\ ${ }^{1}$ State Key Laboratory of Synthetical Automation for Process Industries, Northeastern University, \\ Shenyang, China, 110819 \\ ${ }^{a}$ cxliu@mail.neu.edu.cn
}

Keywords: Coordination operation management, Closed-loop optimization, Plant-wide control, Unified data exchange model.

\begin{abstract}
The cooperative control of technical indices of various unit processes is an essential operation management process to gradually achieve wide-area optimization of the production process. There are so many factors, and the adjustment of which is coupled. This needs to be taken into account when to perform the operation control of a unit process index. To achieve effective and timely structuring of operation management, the framework of a closed-loop optimization system for enhancing the operation management ability of plant-wide production process is proposed. The structure and functions of the framework are introduced in detail. In the end, this paper presents a development meta-system to support the implementation of the proposed scheme and the application of the framework.
\end{abstract}

\section{Introduction}

A whole production process is usually composed of several unit processes connected sequentially. Each unit process performs a particular function in the whole production line and achieves its own technical indices which represent the efficiency, quality and quantity of the local process. It is well known that the local optimization of unit process in the whole production process does not guarantee global optimization of the whole plant.

The cooperative control of operation management implementing economically optimal operating policy is currently gaining attention in the operation field of industrial processed [1-3]. The goal is to find a set of controlled cooperative operations so as to indirectly lead to optimal operation with acceptable loss. Since each unit technical indices are determined by the overall plant behaviors, it is necessary to take a plant-wide perspective. However, it is hard to establish the global operation model for each local index control process, because the relationships between the physical mechanisms and devices disturbances cannot be described clearly. The existing reported research such as [3-5] involves the combination of data-driven and mechanism model which makes use of the process data such as operational data, quality data, device information, etc. Those papers proposed algorithms or special applications with the weakness of lacking a framework of effective realization or practice. It is obvious that the increment complex of factors is needed to be taken into account when to perform an index operation which highlights the need for a better optimization system for enhancing the ability of the operation management. This is because it is too difficultly, if not impossible, to efficiently operate such a complex situation without an effective computer-aided system.

Therefore, this paper focuses on proposing a conceptual model of an optimization framework towards operation management system. Through introducing the concept of closed-loop, the paper suggests a function structure of the framework, and proves its feasibility with a development experiment of a meta-system. This paper will be structured as follows. In section II the analysis of the framework is exposed and the proposed architecture design is provided in section III. The followed section IV shows an application sample to demonstrate the development platform's versatility and practical value. Finally, the conclusion is presented in section $\mathrm{V}$. 


\section{Analysis of System Framework}

In order to address the system needs mentioned above, three key points should be solved [6, 7]: (1) the system should encapsulate useful data information of each unit process to hide the difference from operations, which means that the system should provide a unified global representation of data independent of local unit process; (2) the structure of the system framework should be scalable to support the adaptation of using different type of optimization algorithms for coordinating operation management; (3) the possibility of reusing and replacing system components existed or being developed.

The operation information of organizing and index control to be coordinated is done here by collecting and forming a unified data model, including the real production environment information, the required plant-wide goals, and the operations, etc. It is useful to reduce the time of getting data that are needed by different components or algorithms. Meanwhile, the system based on the proposed framework is responsible for executing those tasks which are called coordination and sub-cooperation, and the unified global data model plays the important role of exchanging data among system components. By overseeing the plant-wide performance, the optimization system for operation management dynamically tunes the unit process goals and indirectly coordinates the wide-area operations. Since the system should be updatable or extendable, it is worthy of paying more attention to the adaptability and extendibility of the system framework.

\section{Design of Closed-loop Optimization Framework for Operation Management}

The overall architecture chart of the proposed framework, considering the above abstractions, is illustrated in Figure 1. According to the architecture of the framework, the design is constructed from unified data representation model layer, closed-loop operation optimization layer and component module layer.

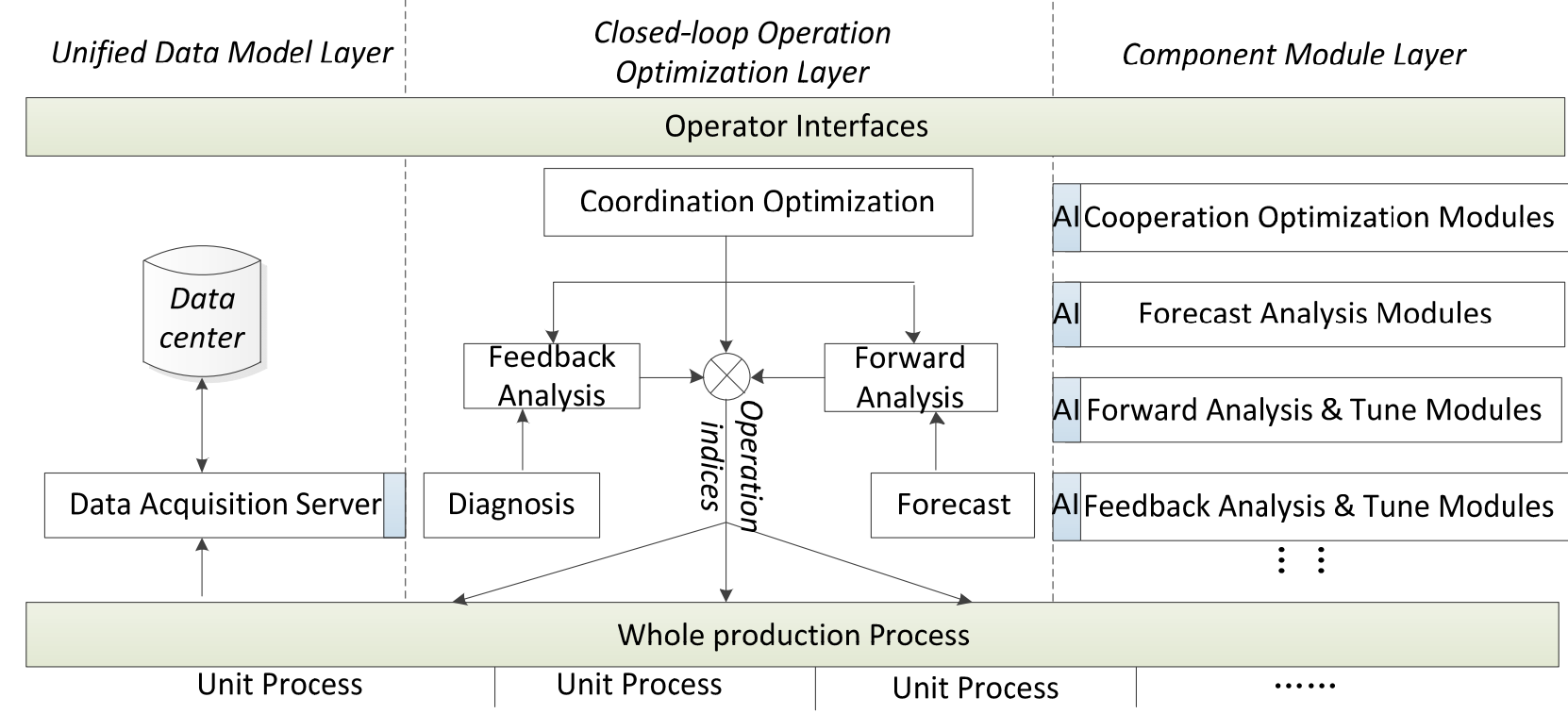

Fig. 1 Conceptual diagram of the proposed framework

A. Unified data representation model layer

The unified data model is of great importance since it is the key to dramatically reducing information access time, especially when exchanging heterogeneous data formats which are traditionally used in different unit process. The data model is composed by three parts: the target part includes the required goals; the operation part includes the operations of manual tuned and the optimization system providing operation time, devices states, materiel information, and etc.; the actual part includes the current process data, sampling time and manual analysis data. Encapsulating those relative data into a unified modal makes it easy to validate the effectiveness and the completeness of the production data, and it can avoid different modules accessing original data 
repeatedly. One structure of the data model could be a multi-layer tree form, which have been putted into practice application. All data are stored into a data center definitely.

B. Closed-loop operation optimization layer

Introducing the concept of closed-loop to operation optimization is obviously different from the traditional operation management, which is used to execute once or local-aimed reported in [7]. The proposed layer includes coordination optimization module overseeing the plant-wide performance. Its output does not transfer to sub-unit process immediately, but use forecast module to analyze the effects of operations and tune them if necessary. The history data from data acquisition server is used to diagnose the fault and the feedback analysis module is executed similarly. Finally, the operation goals of each unit process can be distributed, and it has used the information getting from past and forecast future circularly and formulaically. The data and algorithm module come from data layer and module layer separately.

C. Component module layer

The previous layer constructs a closed-loop framework of the operation optimization and this layer provides an adapter interface (AI) for independent modules with different function. Each module may use a private pattern of data and configuration. The AI wraps the input/output, the logic route and the operation context. With the adapter interface those components can communicate and exchange necessary information, and obtain the same protocols within the whole framework. This design can also support replace and update module compatible with AI independently, which improves the adaptability and extendibility of the system framework. The end-user can develop one module with special function and accumulate modules continuously. User can also select or replace modules according to different production condition. This design improves the extendibility of the framework.

\section{Application Sample}

An optimization meta-system of operation management for the whole production process of ore dressing is developed on the basis of the proposed framework. XML and MEF technologies are used to realize the encapsulating unified data model and the loosely-couple plugin-like functional module which composing this loosely-couple system.

Here is a snapshot of the system which implemented representative functions. The DAS shown in the figure is a reference to the data acquisition server. The meta-system covers the whole key point analyzed above. Furthermore, system includes other essential modules which are not described in detail here because of the limitation of pages. Operators can click the colorful block to execute the selected function sequentially and generate the cooperative operation guide of indices. Experiments of system application prove the friendliness of this optimization framework and the efficiency of the design and the development of a new system.

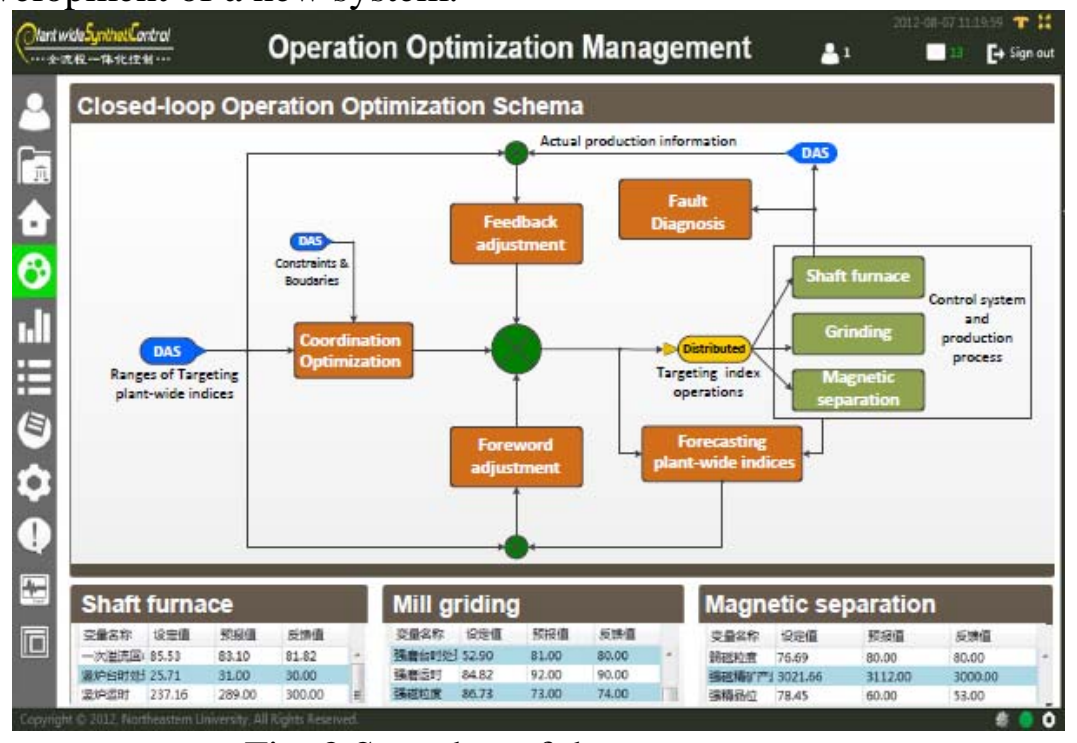

Fig. 2 Snapshot of the meta-system 


\section{Conclusion}

In this paper, we have proposed a closed-loop framework of optimization system for the operation management in a production process, based on which a system with those characteristics mentioned above can be implemented effectively and timely. By using the history and the predication information circularly and formulaically within the framework automatically, local operators can realize the coordination of other unit process, which leads to global optimization of the production process. However, the operation management of the production includes many other factors, and the proposed framework focuses on the key factor of cooperation and providing an applicable framework for enhancing the ability of operation management. The adaptive data mining schema remains to be researched in the further, which is to be integrated into the proposed framework, for operation data stored in data center.

\section{Acknowledgment}

The research was supported by National Basic Research Program of China (973 Program) (2009CB320604).

\section{References}

[1] E. Sebastian: Feedback Control for Optimal Process Operation, Journal of Process Control, 17 (2007), p. 203-219.

[2] S. Sigurd: Plantwide Control: The Search for the Self-Optimizing Control Structure, Journal of Process Control, 10 (2002), p. 487-507.

[3] W. Günter, L. Pu: Planning and optimization of dynamic plant operation, Applied Thermal Engineering, 20 (2000), p. 1393-1407.

[4] D. Jinliang, C. Tianyou, W. Hong, C. Xinkai: Knowledge-based Global Operation of Mineral Processing Under Uncertainty, Industrial Informatics, IEEE Transactions on, 2012 (accepted).

[5] C. Tianyou, D. Jinliang, W. Fenghua: Hybrid intelligent control for optimal operation of shaft furnace roasting process, Control Engineering Practice, 19 (2011) , p. 264-275.

[6] W. Jan, W. Heinz: A security architecture integrated co-operative engineering platform for organised model exchange in a Digital Factory environment, Computers in Industry, 56 (2005), p. 347-360.

[7] C. Tianyou, D. Jinliang: Integrated automation system for hematite ores processing and its applications, Measurement and Control, 39 (2006), p. 140-146. 